\title{
On the Fundamental Tradeoff Between Watermark Detection Performance and Robustness Against Sensitivity Analysis Attacks
}

\author{
Maha El Choubassi ${ }^{a}$ and Pierre Moulin ${ }^{b}$ \\ University of Illinois, Beckman Institute and ECE Department, \\ 405 N. Mathews, Urbana, IL 61801, USA
}

\begin{abstract}
Despite their popularity, spread spectrum techniques have been proven to be vulnerable to sensitivity analysis attacks. Moreover, the number of detection operations needed by the attacker to estimate the watermark is generally linear in the size of the signal available to him. This holds not only for a simple correlation detector, but also for a wide class of detectors. Therefore there is a vital need for more secure detection methods. In this paper, we propose a randomized detection method that increases the robustness of spread spectrum embedding schemes. However, this is achieved at the expense of detection performance. For this purpose, we provide a framework to study the tradeoff between these two factors using classical detection-theoretic tools: large deviation analysis and Chernoff bounds. To gain more insight into the practical value of this framework, we apply it to image signals, for which "good" statistical models are available.
\end{abstract}

Keywords: Watermarking, spread spectrum, sensitivity analysis attacks, randomized detection, security, generalized Gaussian hosts

\section{INTRODUCTION}

By having unlimited access to a watermark detector, an attacker can estimate and "remove" the watermark if the embedding scheme is the spread spectrum scheme (SS). ${ }^{1-8}$ Such attacks have been called "sensitivity analysis attacks". The number of detection operations required for this purpose is linear in the size of the signal available to the attacker, not only for a correlation detector, but for a wide class of detectors. ${ }^{3}$

In this paper, we propose a new detection technique that enhances the robustness of SS embedding schemes against sensitivity analysis attacks. This is realized by introducing substantial randomness into the detector. Linnartz and van Dijk, ${ }^{5}$ and Venkatesan and Jakubowski ${ }^{8}$ also used "randomized" detection to increase security. However, this is achieved at the expense of detection performance, a fact that is not emphasized by these authors. Note that the randomized detection used here is not at all the same as optimal detection in a Neyman-Pearson or minimax sense. ${ }^{9}$ Actually, the randomized detector used by Linnartz and van Dijk, ${ }^{5}$ Venkatesan and Jakubowski, ${ }^{8}$ and here is a mismatched one,${ }^{10}$ and the gain in watermark security is obtained only by sacrificing some detection performance.

For this purpose, we provide a framework to study the tradeoff between these two factors. We construct a large class (whose cardinality is exponential in $n$, the length of the watermark) of provably "good" detection functions. Any time the detector is tested, it randomly chooses a detection function from that class. Our detection method not only improves the security of the watermarking system against sensitivity analysis attacks, but also efficiently lends itself to performance analysis by the application of large-deviation tools.

The organization of this paper is as follows. In Section 2, we describe our proposed detector. In Section 3, we analyze the reliability of our detection method and obtain upper and lower bounds on the error exponents. In Section 4, randomized detectors are generated for images that are modeled as generalized Gaussian signals, ${ }^{11}$ and the theory is illustrated with simulation results. Finally, we conclude in Section 5.

Further author information: (Send correspondence to Maha El Choubassi)

E-mail: cel@ifp.uiuc.edu, Telephone: 12172441089

E-mail: moulin@ifp.uiuc.edu 


\section{SCHEME DESCRIPTION}

The detection problem can be modeled as a binary hypothesis test. There are two hypotheses, $H_{0}$ and $H_{1}$. The watermark $\mathbf{w}=\left(w_{1}, w_{2}, \ldots, w_{n}\right)$ is fixed and known to the detector. For simplicity of the exposition, let the watermark be binary, i.e., $\mathbf{w} \in\{-1,1\}^{n}$. Also, assume that $n$ is even and that $\frac{n}{2}$ of the samples are marked with +1 , while the other half are marked with -1 . The signal input to the detector is $\mathbf{Y}=\left(Y_{1}, Y_{2}, \ldots, Y_{n}\right)$. $H_{0}$ is the hypothesis that $\mathbf{w}$ is not embedded in $\mathbf{Y}$ while $H_{1}$ indicates that $\mathbf{w}$ is embedded

$$
\begin{cases}H_{0}: & \mathbf{Y}=\mathbf{X} \\ H_{1}: & \mathbf{Y}=\mathbf{X}+\mathbf{w}\end{cases}
$$

where $\mathbf{X}=\left(X_{1}, X_{2}, \ldots, X_{n}\right)$ is the host signal. The samples $X_{i}$ are assumed to be independent and identically distributed (iid), with probability density function (pdf) $p(x)$. The optimal detector for the problem (1a) is the log likelihood ratio test:

$$
\sum_{i=1}^{n} \ln \frac{p\left(Y_{i}-w_{i}\right)}{p\left(Y_{i}\right)} \underset{H_{0}}{\stackrel{H_{1}}{\gtrless}} \tau .
$$

However, if we fix the test (1b), this detector is vulnerable to sensitivity analysis attacks. Specifically, the attacker will seek a perturbation $\mathbf{z}$ and a forgery $\mathbf{y}=\mathbf{x}+\mathbf{w}+\mathbf{z}$ such that the detector outputs $H_{0}$. Therefore, there is a need for a randomized detection statistic.

In our new proposed method, we choose a small number $K$ (say 2) and $K$ pdf's $q_{k}(x)$ that are mismatched (in a controllable way) to the pdf $p(x)$. To each $q_{k}$ corresponds a log likelihood ratio

$$
f_{k}(y, w)=\ln \frac{q_{k}(y-w)}{q_{k}(y)} .
$$

for the detector. The "goodness" of each $q_{k}$ is measured by the information divergence (or Kulback-Leibler divergence) between $p($.$) and q_{k}($.$) , defined as$

$$
D\left(p \| q_{k}\right)=\int_{-\infty}^{+\infty} p(x) \ln \frac{p(x)}{q_{k}(x)} d x .
$$

This measure is equal to zero for a perfect match $\left(q_{k}=p\right)$. We assume that the pdf's $p$ and $q_{1}, q_{2}, \ldots, q_{K}$ have common support.

The signal domain $\{1,2, \ldots, n\}$ is partitioned into $K$ subsets $\Omega_{k}, 1 \leq k \leq K$, each of fixed length $n_{k}=\lambda_{k} n$, assumed to be even. Let $\Omega=\left\{\Omega_{1}, \ldots, \Omega_{K}\right\}$. Observe that $\lambda=\left\{\lambda_{k}, 1 \leq k \leq K\right\}$ may be viewed as a probability distribution. Each subset $\Omega_{k}$ is selected such that exactly half of the samples in $\Omega_{k}$ are marked with +1 , while the other half are marked with -1 . Hence each time it is probed, the detector, who knows the watermark $\mathbf{w}$, picks a random collection of such subsets and computes the decision statistic

$$
T(\mathbf{Y}, \mathbf{w} \mid \Omega)=\sum_{k=1}^{K} \sum_{i \in \Omega_{k}} f_{k}\left(Y_{i}, w_{i}\right) .
$$

In order to generate $\left\{\Omega_{1}, \Omega_{2}, \ldots, \Omega_{K}\right\}$, we partition the set $\{1, \ldots, n\}$ into two subsets $Z_{1, \mathbf{w}}=\left\{i: w_{i}=1\right\}$ and $Z_{2, \mathbf{w}}=\left\{i: w_{i}=-1\right\}$, where $\left|Z_{1, \mathbf{w}}\right|=\left|Z_{2, \mathbf{w}}\right|=\frac{n}{2}$. For each subset $\Omega_{k}$, we randomly pick half of its elements, $\frac{n_{k}}{2}$, from $Z_{1, \mathbf{w}}$. The number of all such possible subsets is essentially equal to $2^{\frac{n}{2} H(\lambda)}$, where $H(\lambda)=-\sum_{k=1}^{K} \lambda_{k} \log _{2} \lambda_{k}$ denotes the entropy of the probability distribution $\lambda$. We pick the remaining $\frac{n_{k}}{2}$ elements of $\Omega_{k}$ from $Z_{2, \mathbf{w}}$ in the same way. Therefore, the number of all possible partitions $\left\{\Omega_{1}, \Omega_{2}, \ldots, \Omega_{K}\right\}$ is exponential in $n$ :

$$
2^{n H(\lambda)}=2^{\frac{n}{2} H(\lambda)} \times 2^{\frac{n}{2} H(\lambda)} .
$$


The pdf for the observation vector $\mathbf{y}$ is given by

$$
\begin{aligned}
& p_{\mathbf{Y}, 0}\left(y_{1}, \ldots, y_{n}\right)=\prod_{i=1}^{n} p\left(y_{i}\right) \quad \text { under } H_{0}, \\
& p_{\mathbf{Y}, 1}\left(y_{1}, \ldots, y_{n}\right)=\prod_{i=1}^{n} p\left(y_{i}-w_{i}\right) \quad \text { under } H_{1},
\end{aligned}
$$

The mismatched pdf is defined as

$$
\begin{aligned}
& q_{\mathbf{Y}, 0}\left(y_{1}, \ldots, y_{n}\right)=\prod_{k=1}^{K} \prod_{i \in \Omega_{k}} q_{k}\left(y_{i}\right) \quad \text { under } H_{0}, \\
& q_{\mathbf{Y}, 1}\left(y_{1}, \ldots, y_{n}\right)=\prod_{k=1}^{K} \prod_{i \in \Omega_{k}} q_{k}\left(y_{i}-w_{i}\right) \quad \text { under } H_{1} .
\end{aligned}
$$

In other words, this is equivalent to assuming (for detection purposes) that the host samples in subset $\Omega_{k}$ follow the distribution $q_{k}(x)$ for all $k \in\{1, \ldots, K\}$.

Note that in Venkatesan and Jakubowski's paper, ${ }^{8}$ the detection statistic is the median of the correlation statistic calculated over several randomly selected subsets of the signal. In this paper, the test statistic in (2) is a mixture of several "good" statistics computed over the randomized subsets $\Omega_{k}$.

The security enhancement comes from the randomness of the sets $\Omega_{k}$. By randomly selecting subsets according to the probability distribution $\lambda=\left(\lambda_{1}, \lambda_{2}, \ldots, \lambda_{K}\right)$, we generate an exponentially large $\left(2^{n H(\lambda)}\right)$ class of randomized test statistics. For comparison, Linnartz and van Dijk ${ }^{5}$ increase the security of the watermarking scheme by randomizing the detection threshold $\tau$ which belongs to an uncertainty interval $[A, B]$. Let $\theta=\tau$ be the randomization parameter used resulting in the test statistic $T(\mathbf{Y}, \mathbf{w} \mid \theta \in \Theta)$. Obviously the size of parameter space $\Theta$ in our scheme is much larger. Therefore, the security improvements are likely to be more significant.

\section{DETECTION RELIABILITY}

In this section, the performance of the new detection method is studied. The construction of our test statistic is amenable to a thorough performance analysis. Since it is generally infeasible to compute the probability of error $\left(P_{e}\right)$ analytically, large deviation techniques and Chernoff bounds ${ }^{9}$ are used. These bounds are of the form

$$
P_{e} \leq \exp \left\{-n \mu^{*}\right\}
$$

where $\mu:[0, \infty) \rightarrow \mathbb{R}$ is the large deviation function

$$
\mu(s) \triangleq-\frac{1}{n} \ln \mathbb{E}[\exp (s T(\mathbf{X}, \mathbf{w} \mid \Omega))], \quad s \geq 0,
$$

and $\mu^{*}$ is the maximum of this function realized at $s^{*}$

$$
\mu^{*}=\mu\left(s^{*}\right)=\max _{s \geq 0} \mu(s) .
$$

By Cramer's theorem, the inequality (3) is tight on the exponential scale:

$$
\lim _{n \rightarrow \infty} \frac{-1}{n} \ln P_{e}=\mu^{*} .
$$

However, care must be taken since the detector is a mismatched detector. ${ }^{10}$ In this section, upper and lower bounds on the performance error are derived in order to quantify the advantages and limitations of our detector. 


\subsection{Large Deviation Functions}

The analysis is made for a given watermark $\mathbf{w}$ known to the detector. Referring to the statistical model (1a), we will use the shorthands $P_{0}(\cdot)$ in place of $P\left(\cdot \mid H_{0}\right)$, and $\mathbb{E}_{0}[\cdot]$ in place of $\mathbb{E}\left[\cdot \mid H_{0}\right]$. Similarly, we define $P_{1}(\cdot)$ and $\mathbb{E}_{1}[\cdot]$.

The fact that the host signal samples, $X_{i} 1 \leq i \leq n$, are iid, together with the assumptions made about the subsets $\Omega_{k}$ and the watermark $\mathbf{w}$, imply that the distribution of the detection statistic of (2) is independent of the specific partition $\Omega=\left\{\Omega_{1}, \Omega_{2}, \ldots, \Omega_{K}\right\}$. Therefore, the probability of false alarm is defined as

$$
P_{F}=P_{0}(T(\mathbf{Y}, \mathbf{w} \mid \Omega)>\tau) .
$$

Substituting (2) into the above expression, we obtain

$$
P_{F}=P_{0}\left(\exp \left(s \sum_{k=1}^{K} \sum_{i \in \Omega_{k}} f_{k}\left(Y_{i}, w_{i}\right)\right)>e^{s \tau}\right), \quad \forall s>0 .
$$

Note that we have $f_{k}\left(Y_{i}, w_{i}\right)=\ln \frac{q_{k}\left(Y_{i}-w_{i}\right)}{q_{k}\left(Y_{i}\right)}$ and that by our assumption below $(1 \mathrm{~d})$, we obtain $P_{0}\left(q_{k}\left(Y_{i}\right)=0\right)=0$. Markov's inequality yields

$$
P_{F} \leq e^{-s \tau} \mathbb{E}_{0}\left[\exp \left(s \sum_{k=1}^{K} \sum_{i \in \Omega_{k}} f_{k}\left(Y_{i}, w_{i}\right)\right)\right], \quad \forall s>0 .
$$

Noting that $\mathbf{Y}=\mathbf{X}$ under $\mathbf{H}_{0}$ and that the signal samples $X_{1}, X_{2}, \ldots, X_{n}$ are iid, (5) becomes

$$
P_{F} \leq e^{-s \tau} \Pi_{k=1}^{K} \mathbb{E}\left[\exp \left(s \sum_{i \in \Omega_{k}} f_{k}\left(X_{i}, w_{i}\right)\right)\right], \quad \forall s>0 .
$$

However, note that

$$
\mathbb{E}\left[\exp \left(s f_{k}\left(X_{i}, w_{i}\right)\right)\right]=\mathbb{E}\left[\exp \left(s f_{k}\left(X_{1}, w_{i}\right)\right)\right], \quad \forall i \in \Omega_{k} .
$$

Each subset $\Omega_{k}$ has $\frac{n_{k}}{2}$ of its samples marked with +1 , and the other $\frac{n_{k}}{2}$ samples marked with -1 . This assumption together with (6) and (7) yield

$$
P_{F} \leq \exp \left\{n\left(-s \tau_{0}+\sum_{k=1}^{K} \frac{n_{k}}{2 n}\left(\ln \mathbb{E}\left[\exp \left(s f_{k}\left(X_{1}, 1\right)\right)\right]+\ln \mathbb{E}\left[\exp \left(s f_{k}\left(X_{1},-1\right)\right)\right]\right)\right)\right\}, \quad \forall s>0,
$$

where $\tau_{0}=\frac{\tau}{n}$. Noticing that $\lambda_{k}=\frac{n_{k}}{n}$, the large deviation bound on the probability of false alarm is given by

$$
\begin{aligned}
P_{F} & \leq \exp \left(-n E_{F}\left(s, \tau_{0}\right)\right), \quad \forall s>0, \\
E_{F}\left(s, \tau_{0}\right) & =s \tau_{0}-\sum_{k=1}^{K} \frac{\lambda_{k}}{2}\left(\ln \mathbb{E}\left[\exp \left(s f_{k}\left(X_{1}, 1\right)\right)\right]+\ln \mathbb{E}\left[\exp \left(s f_{k}\left(X_{1},-1\right)\right)\right]\right), \quad \forall s>0 .
\end{aligned}
$$

Similarly, the probability of miss is defined as

$$
P_{M}=P_{1}(T(\mathbf{Y}, \mathbf{w} \mid \Omega) \leq \tau) .
$$

The large deviations upper bound on $P_{M}$ is given by

$$
\begin{aligned}
P_{M} & \leq \exp \left(-n E_{M}\left(s, \tau_{0}\right)\right), \quad \forall s>0 \\
E_{M}\left(s, \tau_{0}\right) & =-s \tau_{0}-\sum_{k=1}^{K} \frac{\lambda_{k}}{2}\left(\ln \mathbb{E}\left[\exp \left(-s f_{k}\left(X_{1}+1,1\right)\right)\right]+\ln \mathbb{E}\left[\exp \left(-s f_{k}\left(X_{1}-1,-1\right)\right)\right]\right), \quad \forall s>0 .
\end{aligned}
$$




\subsection{Exponential Convergence of the Average Probability of Error}

Obviously, our test statistic $T(\mathbf{y}, \mathbf{w} \mid \Omega)$ is not the log likelihood ratio corresponding to the real probability distribution on $\mathbf{X}$. Hence, it is not the optimal one, and we have a mismatched detector. In his paper, ${ }^{10}$ Kazakos derives the performance of such detectors. The exponents of the probabilities of false alarm and miss may not be positive, i.e., $P_{F}$ and $P_{M}$ may not decay to zero exponentially with increasing $n$. Kazakos provides a necessary and sufficient condition for exponential convergence. However, this condition is given for the case when the observations are iid under both hypotheses $H_{0}$ and $H_{1}$, and the mismatched detector also assumes iid observations under $H_{0}$ and $H_{1}$. In this section, we derive the necessary and sufficient condition for exponential decay of the probability of error with $n$ in our set up: independent observations but not necessarily identically distributed.

For the following analysis to be valid, we need the first and the second derivatives with respect to $s$ of the large deviation bounds $E_{F}\left(s, \tau_{0}\right)$ and $E_{M}\left(s, \tau_{0}\right)$ given in (8) and (10). A sufficient condition ${ }^{10}$ for these derivatives to exist, be finite and be computable by interchanging the expectation with the differentiation is given by

$$
\mathbb{E}\left[\frac{q_{k}\left(X_{1}-1\right)}{q_{k}\left(X_{1}\right)}\right]<\infty \quad \text { and } \quad \mathbb{E}\left[\frac{q_{k}\left(X_{1}+1\right)}{q_{k}\left(X_{1}\right)}\right]<\infty, \quad \forall k \in\{1, \ldots, K\} .
$$

Note that from (1c) we have

$$
\exp \left(s f_{k}(y, w)\right)=\left(\frac{q_{k}(y-w)}{q_{k}(y)}\right)^{s} .
$$

Therefore, the large deviation exponents in (9) and (11) are rewritten as

$$
\begin{aligned}
& E_{F}\left(s, \tau_{0}\right)=s \tau_{0}-\sum_{k=1}^{K} \frac{\lambda_{k}}{2}\left(\ln \mathbb{E}\left[\left(\frac{q_{k}\left(X_{1}-1\right)}{q_{k}\left(X_{1}\right)}\right)^{s}\right]+\ln \mathbb{E}\left[\left(\frac{q_{k}\left(X_{1}+1\right)}{q_{k}\left(X_{1}\right)}\right)^{s}\right]\right), \quad \forall s>0, \\
& E_{M}\left(s, \tau_{0}\right)=-s \tau_{0}-\sum_{k=1}^{K} \frac{\lambda_{k}}{2}\left(\ln \mathbb{E}\left[\left(\frac{q_{k}\left(X_{1}-1\right)}{q_{k}\left(X_{1}\right)}\right)^{s}\right]+\ln \mathbb{E}\left[\left(\frac{q_{k}\left(X_{1}+1\right)}{q_{k}\left(X_{1}\right)}\right)^{s}\right]\right), \quad \forall s>0 .
\end{aligned}
$$

Under assumption (12), the first derivatives with respect to $s$ of $E_{F}\left(s, \tau_{0}\right)$ and $E_{M}\left(s, \tau_{0}\right)$ are evaluated at $s=0$ as

$$
\begin{aligned}
\left.\frac{d}{d s} E_{F}\left(s, \tau_{0}\right)\right|_{s=0} & =\tau_{0}-\sum_{k=1}^{K} \frac{\lambda_{k}}{2}\left(\mathbb{E}\left[\ln \left(\frac{q_{k}\left(X_{1}-1\right)}{q_{k}\left(X_{1}\right)}\right)\right]+\mathbb{E}\left[\ln \left(\frac{q_{k}\left(X_{1}+1\right)}{q_{k}\left(X_{1}\right)}\right)\right]\right), \\
\left.\frac{d}{d s} E_{M}\left(s, \tau_{0}\right)\right|_{s=0} & =-\tau_{0}-\sum_{k=1}^{K} \frac{\lambda_{k}}{2}\left(\mathbb{E}\left[\ln \left(\frac{q_{k}\left(X_{1}-1\right)}{q_{k}\left(X_{1}\right)}\right)\right]+\mathbb{E}\left[\ln \left(\frac{q_{k}\left(X_{1}+1\right)}{q_{k}\left(X_{1}\right)}\right)\right]\right) .
\end{aligned}
$$

For any two pdf's $r(\cdot)$ and $h(\cdot)$, we have

$$
\begin{aligned}
\mathbb{E}\left[\ln \left(\frac{r(X)}{h(X)}\right)\right] & =\mathbb{E}\left[\ln \left(\frac{p(X)}{h(X)}\right)\right]-\mathbb{E}\left[\ln \left(\frac{p(X)}{r(X)}\right)\right] \\
& =D(p \| h)-D(p \| r) .
\end{aligned}
$$

We define the shifted pdf's $q_{k}(x-1)$ and $q_{k}(x+1)$ for each $k \in\{1, \ldots, K\}$ as

$$
q_{k, 1}(x):=q_{k}(x-1) \quad \text { and } \quad q_{k,-1}(x):=q_{k}(x+1) .
$$

With this notation, (15) and (16) become

$$
\begin{aligned}
\left.\frac{d}{d s} E_{F}\left(s, \tau_{0}\right)\right|_{s=0} & =\tau_{0}-\sum_{k=1}^{K} \frac{\lambda_{k}}{2}\left(2 D\left(p \| q_{k}\right)-D\left(p \| q_{k, 1}\right)-D\left(p \| q_{k,-1}\right)\right), \\
\left.\frac{d}{d s} E_{M}\left(s, \tau_{0}\right)\right|_{s=0} & =-\tau_{0}-\sum_{k=1}^{K} \frac{\lambda_{k}}{2}\left(2 D\left(p \| q_{k}\right)-D\left(p \| q_{k, 1}\right)-D\left(p \| q_{k,-1}\right)\right) .
\end{aligned}
$$


Moreover, the second derivatives of $E_{F}\left(s, \tau_{0}\right)$ and $E_{M}\left(s, \tau_{0}\right)$ with respect to $s$ are equal and are given by

$$
\begin{aligned}
\frac{d^{2}}{d s^{2}} E_{F}\left(s, \tau_{0}\right)=\frac{d^{2}}{d s^{2}} E_{M}\left(s, \tau_{0}\right) & =-\sum_{k=1}^{K} \frac{\lambda_{k}}{2}\left(\frac{\mathbb{E}\left[A_{k, 1} B_{k, 1}^{2}\right] \mathbb{E}\left[A_{k, 1}\right]-\mathbb{E}\left[A_{k, 1} B_{k, 1}\right]^{2}}{\mathbb{E}\left[A_{k, 1}\right]^{2}}\right. \\
& \left.+\frac{\mathbb{E}\left[A_{k,-1} B_{k,-1}^{2}\right] \mathbb{E}\left[A_{k,-1}\right]-\mathbb{E}\left[A_{k,-1} B_{k,-1}\right]^{2}}{\mathbb{E}\left[A_{k,-1}\right]^{2}}\right), \quad \forall s>0,
\end{aligned}
$$

where $A_{k, j}$ and $B_{k, j}$ are defined for $j \in\{-1,1\}$ as

$$
\begin{aligned}
A_{k, j} & =\left(\frac{q_{k}\left(X_{1}-j\right)}{q_{k}\left(X_{1}\right)}\right)^{s} \geq 0, \quad \forall s>0, \\
B_{k, j} & =\ln \left(\frac{q_{k}\left(X_{1}-j\right)}{q_{k}\left(X_{1}\right)}\right) .
\end{aligned}
$$

For each $k \in\{1, \ldots, K\}$ and $j \in\{-1,1\}$, we have

$$
\begin{aligned}
\left|\mathbb{E}\left[A_{k, j} B_{k, j}\right]\right| & \leq \mathbb{E}\left[\left|A_{k, j}\right|\left|B_{k, j}\right|\right], \\
& =\mathbb{E}\left[\left(\sqrt{A_{k, j}}\left|B_{k, j}\right|\right) \sqrt{A_{k, j}}\right] \\
& \leq \sqrt{\mathbb{E}\left[A_{k, j} B_{k, j}^{2}\right]} \sqrt{\mathbb{E}\left[A_{k, j}\right]}
\end{aligned}
$$

where the last line follows from the Cauchy-Schwarz inequality.

If $q_{k}\left(X_{1}-j\right)=q_{k}\left(X_{1}\right)$ almost everywhere with respect to the probability measure induced by $p(\cdot)$, equality will be achieved in the above expression, and if this is true for all $k \in\{1, \ldots, K\}$ and each $j \in\{-1,1\}$, the second derivative in (20) is equal to zero. However, this is not true for all the probability distributions of interest, and the second derivatives with respect to $s$ of $E_{F}\left(s, \tau_{0}\right)$ and $E_{M}\left(s, \tau_{0}\right)$ are strictly negative. Hence, $E_{F}\left(s, \tau_{0}\right)$ and $E_{M}\left(s, \tau_{0}\right)$ are strictly concave functions of $s$ with their first order derivatives evaluated at $s=0$ given in (18) and (19). Moreover, the functions $E_{F}\left(s, \tau_{0}\right)$ and $E_{M}\left(s, \tau_{0}\right)$ are zero at $s=0$.

Let $P_{e}$ be the average probability of error. Assume that the two hypotheses $H_{0}$ and $H_{1}$ are equiprobable, then $P_{e}$ is equal to

$$
P_{e}=\frac{1}{2} P_{F}+\frac{1}{2} P_{M}
$$

Owing to the large deviation bounds (8) and (10) on $P_{F}$ and $P_{M}$, we obtain

$$
P_{e} \leq \frac{1}{2} \exp \left(-n E_{F}\left(s, \tau_{0}\right)\right)+\frac{1}{2} \exp \left(-n E_{M}\left(s, \tau_{0}\right)\right) .
$$

From the above equation, it is clear that $P_{e}$ decays exponentially to zero if both $P_{M}$ and $P_{F}$ decay exponentially to zero. This property is guaranteed if and only if there exist a pair $\left(s, \tau_{0}\right) \in(0, \infty) \times(-\infty, \infty)$ satisfying:

$$
E_{F}\left(s, \tau_{0}\right)>0 \text { and } E_{M}\left(s, \tau_{0}\right)>0 .
$$

Since $E_{F}\left(s, \tau_{0}\right)$ and $E_{M}\left(s, \tau_{0}\right)$ are strictly concave functions in $s$ and are zero at $s=0$, the above condition is equivalent to

$$
\left.\frac{d}{d s} E_{F}\left(s, \tau_{0}\right)\right|_{s=0}>0 \text { and }\left.\quad \frac{d}{d s} E_{M}\left(s, \tau_{0}\right)\right|_{s=0}>0 .
$$

Substituting (18) and (19) into the above inequalities, we have

$$
-\left|\tau_{0}\right|-\sum_{k=1}^{K} \frac{\lambda_{k}}{2}\left(2 D\left(p \| q_{k}\right)-D\left(p \| q_{k, 1}\right)-D\left(p \| q_{k,-1}\right)\right)>0 .
$$




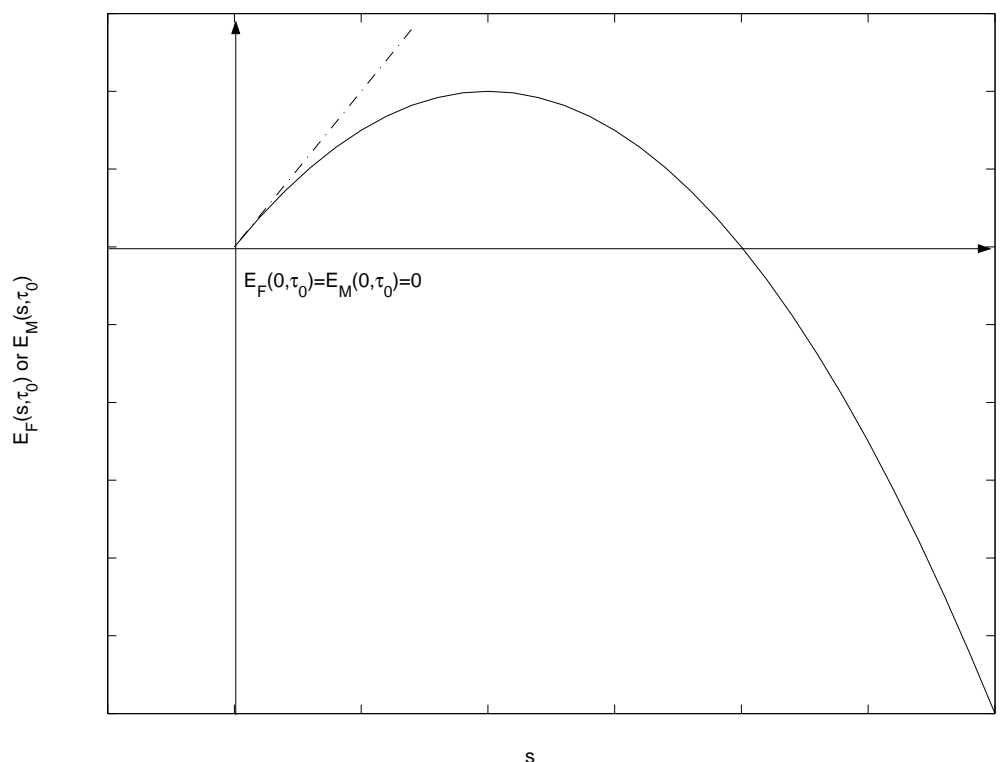

Figure 1. An example of a function with similar properties as $E_{F}\left(s, \tau_{0}\right)$ and $E_{M}\left(s, \tau_{0}\right)$.

If the above inequality is satisfied, there exists a positive number $s_{0}$ such that

$$
E_{F}\left(s, \tau_{0}\right)>0 \quad \text { and } \quad E_{M}\left(s, \tau_{0}\right)>0, \quad \forall s \in\left[0, s_{0}\right]
$$

Please refer to Figure 1 for an illustration.

Consequently, a necessary and sufficient condition for exponential decay of the average probability of error $P_{e}$ is given by

$$
\sum_{k=1}^{K} \frac{\lambda_{k}}{2}\left(2 D\left(p \| q_{k}\right)-D\left(p \| q_{k, 1}\right)-D\left(p \| q_{k,-1}\right)\right)<0 .
$$

If this condition is not satisfied, then for any value of $\tau_{0}$, at least one of the exponents $E_{F}\left(s, \tau_{0}\right)$ or $E_{M}\left(s, \tau_{0}\right)$ is negative for all $s \in(0,+\infty)$. Therefore, this condition is necessary.

Moreover, this condition is sufficient. If the inequality $(\mathrm{C})$ is obeyed, we can choose the threshold $\tau_{0}$ such that (22) is also obeyed.

\subsection{Tightest Bound on $P_{e}$}

In the previous section, we derived the necessary and sufficient condition $(\mathrm{C})$ for exponential convergence to zero of the average probability of error $P_{e}$. This condition is given in terms of the information divergence between the real and the assumed probability distributions. If $(\mathrm{C})$ is satisfied, there is an optimal value for the threshold $\tau_{0}$ that results in the tightest bound on the average probability of error $P_{e}$. In what follows, we derive this value and obtain the corresponding bound on $P_{e}$.

Assuming that the two hypotheses are equiprobable, we know from Section 3.2 that

$$
P_{e} \leq \frac{1}{2} \exp \left(-n E_{F}\left(s, \tau_{0}\right)\right)+\frac{1}{2} \exp \left(-n E_{M}\left(s, \tau_{0}\right)\right)
$$

In order for this bound to be optimal, the two exponents have to be equal, otherwise the worse one, i.e. the smaller one, will dominate the other. Note from (13) and (14) that the sum $E_{F}\left(s, \tau_{0}\right)+E_{M}\left(s, \tau_{0}\right)$ is independent of $\tau_{0}$ and that the value of $\tau_{0}$ that equalizes the exponents is zero. However, we also need to optimize the bound 
over $s$, i.e. we need to maximize $E_{F}(s, 0)=E_{M}(s, 0)$ over $s$. Let $s^{*}$ be the maximizer. The resulting tightest upper bound on $P_{e}$ is

$$
P_{e} \leq \exp \left(n \sum_{k=1}^{K} \frac{\lambda_{k}}{2}\left(\ln \mathbb{E}\left[\left(\frac{q_{k}\left(X_{1}-1\right)}{q_{k}\left(X_{1}\right)}\right)^{s^{*}}\right]+\ln \mathbb{E}\left[\left(\frac{q_{k}\left(X_{1}+1\right)}{q_{k}\left(X_{1}\right)}\right)^{s^{*}}\right]\right)\right) .
$$

\subsection{Upper and Lower Bounds on the Tightest Exponent}

When condition $(\mathrm{C})$ is satisfied, the average probability of error corresponding to the randomized test statistic of (2) decays exponentially to zero. From (23), the rate of decay is

$$
\mu^{*}=-\sum_{k=1}^{K} \frac{\lambda_{k}}{2}\left(\ln \mathbb{E}\left[\left(\frac{q_{k}\left(X_{1}-1\right)}{q_{k}\left(X_{1}\right)}\right)^{s^{*}}\right]+\ln \mathbb{E}\left[\left(\frac{q_{k}\left(X_{1}+1\right)}{q_{k}\left(X_{1}\right)}\right)^{s^{*}}\right]\right) .
$$

On the other hand, consider the case when the $k^{\text {th }}$ test statistic is used for all samples, i.e., $T(\mathbf{y}, \mathbf{w})=$ $\sum_{i=1}^{n} f_{k}\left(y_{i}, w_{i}\right)$. This corresponds to $\Omega_{k}=\{1, \ldots, n\}$ and $\Omega_{j}=\phi, \forall j \neq k$. Let $P_{e, k}$ be the average probability of error for this test. In this case, the condition (C) reduces to

$$
2 D\left(p \| q_{k}\right)-D\left(p \| q_{k, 1}\right)-D\left(p \| q_{k,-1}\right)<0 .
$$

When (25) is satisfied, $P_{e, k}$ decays exponentially to zero, and its large deviations upper bound is given by

$$
\begin{aligned}
P_{e, k} & \leq \exp \left(-n \mu_{k}(s)\right), \quad \forall s>0, \\
\mu_{k}(s) & =-\frac{1}{2}\left(\ln \mathbb{E}\left[\left(\frac{q_{k}\left(X_{1}-1\right)}{q_{k}\left(X_{1}\right)}\right)^{s}\right]+\ln \mathbb{E}\left[\left(\frac{q_{k}\left(X_{1}+1\right)}{q_{k}\left(X_{1}\right)}\right)^{s}\right]\right), \quad \forall s>0 .
\end{aligned}
$$

As stated earlier, the randomized detector consists of a mixture of "good" statistics, i.e., statistics whose probability of error decays to zero as $n$ increases. For this reason, it is natural to require that the pdf's generating these statistics, $q_{k}(\cdot)$ for $k \in\{1, \ldots, K\}$, satisfy (25). Actually, if each pdf $q_{k}(\cdot)$ satisfies $(25)$, then by multiplying $(25)$ by $\lambda_{k}$ and summing over $k \in\{1, \ldots, K\}$, we conclude that condition (C) is also satisfied. Therefore, $P_{e}$ is also guaranteed to decay exponentially to zero, and satisfying (25) for each $k \in\{1, \ldots, K\}$ is a sufficient condition for that.

Under $(25), \mu_{k}(s)$ is a strictly concave function of $s$. It can be maximized to give the tightest upper bound on $P_{e j}$. Let $s_{k}$ be the maximizer of the upper bound on $P_{e, k}$ :

$$
s_{k}=\arg \max _{s>0}\left(\mu_{k}(s)\right), \quad k \in\{1, \ldots, K\} .
$$

Thus, evaluating $\mu_{k}(s)$ at $s_{k}$ in $(26)$, we obtain

$$
\begin{aligned}
& -\frac{1}{2}\left(\ln \mathbb{E}\left[\left(\frac{q_{k}\left(X_{1}-1\right)}{q_{k}\left(X_{1}\right)}\right)^{s^{*}}\right]+\ln \mathbb{E}\left[\left(\frac{q_{k}\left(X_{1}+1\right)}{q_{k}\left(X_{1}\right)}\right)^{s^{*}}\right]\right) \\
\leq \quad & -\frac{1}{2}\left(\ln \mathbb{E}\left[\left(\frac{q_{k}\left(X_{1}-1\right)}{q_{k}\left(X_{1}\right)}\right)^{s_{k}}\right]+\ln \mathbb{E}\left[\left(\frac{q_{k}\left(X_{1}+1\right)}{q_{k}\left(X_{1}\right)}\right)^{s_{k}}\right]\right) .
\end{aligned}
$$

Multiplying the above equation by $\lambda_{k}$ and summing over $k \in\{1, \ldots, K\}$, we obtain

$$
\begin{array}{r}
\quad-\sum_{k=1}^{K} \frac{\lambda_{k}}{2}\left(\ln \mathbb{E}\left[\left(\frac{q_{k}\left(X_{1}-1\right)}{q_{k}\left(X_{1}\right)}\right)^{s^{*}}\right]+\ln \mathbb{E}\left[\left(\frac{q_{k}\left(X_{1}+1\right)}{q_{k}\left(X_{1}\right)}\right)^{s^{*}}\right]\right) \\
\leq \quad-\sum_{k=1}^{K} \frac{\lambda_{k}}{2}\left(\ln \mathbb{E}\left[\left(\frac{q_{k}\left(X_{1}-1\right)}{q_{k}\left(X_{1}\right)}\right)^{s_{k}}\right]+\ln \mathbb{E}\left[\left(\frac{q_{k}\left(X_{1}+1\right)}{q_{k}\left(X_{1}\right)}\right)^{s_{k}}\right]\right),
\end{array}
$$


which is equivalent to:

$$
\mu^{*} \leq \sum_{k=1}^{K} \lambda_{k} \mu_{k}^{*}
$$

where $\mu_{k}^{*}=\mu_{k}\left(s_{k}\right)>0$ is the rate of decay of $P_{e, k}$ to zero.

Hence, we have an upper bound on the performance of the randomized detector. The tightest error exponent $\mu^{*}$ cannot exceed the average of all the individual exponents of the used statistics. This implies that even if the condition $(\mathrm{C})$ is satisfied for all the individual test statistics considered, i.e. (25) is satisfied $\forall k \in\{1, \ldots, K\}$, the probability of error of the randomized test will decay to zero exponentially with increasing $n$ but at a rate slower then the average of the individual rates. This marks the tradeoff between robustness and detection performance. Randomization increases security, but the security improvements come at the expense of a loss in detection performance.

Consequently, having a lower bound on the performance is of high interest since it guarantees that the performance will not be worse than this lower bound. Let $k_{0} \in\{1,2, \ldots, K\}$ be the index of the "worst" test statistic $T_{k 0}(\mathbf{y}, \mathbf{w})$. What we mean by "worst", is that the test results with the minimum tightest error exponent among all other tests $T_{k}(\mathbf{y}, \mathbf{w}), \forall k \in\{1,2, \ldots, K\}$, i.e.,

$$
\mu_{k 0}\left(s_{k 0}\right)=\mu_{k 0}^{*} \leq \mu_{k}^{*} \quad \forall k \in\{1,2, \ldots, K\} .
$$

In the following, we specify a sufficient condition for the randomized detector's error exponent $\mu^{*}$ to be better than the "worst" exponent $\mu_{k 0}^{*}$.

THEOREM 1. If we have

$$
\mu_{k}\left(s_{k 0}\right) \geq \mu_{k 0}^{*}, \quad \forall k \in\{1,2, \ldots, K\}
$$

then the tightest error exponent of the randomized detector is guaranteed to be better or at least same as the "worst" exponent

$$
\mu^{*} \geq \mu_{k 0}^{*} .
$$

Proof: Combining (24) with (26), we conclude that

$$
\mu^{*}=\sum_{k=1}^{K} \lambda_{k} \mu_{k}\left(s^{*}\right) .
$$

However, we know that

$$
\mu^{*}=\max _{s>0} \sum_{k=1}^{K} \lambda_{k} \mu_{k}(s) .
$$

This fact together with (30) results in

$$
\begin{aligned}
\mu^{*} & =\sum_{k=1}^{K} \lambda_{k} \mu_{k}\left(s^{*}\right) \\
& \geq \sum_{k=1}^{K} \lambda_{k} \mu_{k}\left(s_{k 0}\right) \\
& \geq \sum_{k=1}^{K} \lambda_{k} \mu_{k 0}\left(s_{k 0}\right), \quad \text { by }(29) \\
& =\mu_{k 0}\left(s_{k 0}\right), \quad \text { since } \sum_{k=1}^{K} \lambda_{k}=1 .
\end{aligned}
$$

Therefore, we have $\mu^{*} \geq \mu_{k 0}^{*}$. 


\section{GENERALIZED GAUSSIAN HOSTS}

It is common to model the DCT coefficients of an image as iid random variables distributed according to the generalized Gaussian distribution ${ }^{11}$ (GGD). We will use this model in order to generate the "good" statistics for our randomized test.

We will assume that the host signal $\mathbf{X}$ is composed of iid samples $X_{1}, X_{2}, \ldots, X_{n}$ distributed according to a Laplacian distribution with variance $2 b^{2}$ :

$$
p(x)=\frac{1}{2 b} \exp \left(-\frac{|x|}{b}\right) .
$$

In order to get the $K$ "good" statistics, we will consider $K$ GGD distributions:

$$
q_{k}(x)=\frac{\beta_{k}}{2 \alpha_{k} \Gamma\left(\frac{1}{\beta_{k}}\right)} \exp \left(-\left|\frac{x}{\alpha_{k}}\right|^{\beta_{k}}\right) .
$$

where $\left(\beta_{k}>0\right)$ is the exponent of the GGD.

The parameters $\alpha_{k}$ and $\beta_{k}$ are selected such that the variance of the $k^{\text {th }}$ GGD distribution is the same as the variance of the host samples

$$
\alpha_{k}^{2} \frac{\Gamma\left(\frac{3}{\beta_{k}}\right)}{\Gamma\left(\frac{1}{\beta_{k}}\right)}=2 b^{2} .
$$

This is true for a given exponent $\beta_{k}$ if we set

$$
\alpha_{k}=\sqrt{2 b^{2} \frac{\Gamma\left(\frac{1}{\beta_{k}}\right)}{\Gamma\left(\frac{3}{\beta_{k}}\right)}} .
$$

From (1c) and (31), we obtain the $k^{\text {th }}$ statistic $f_{k}\left(y_{i}, w_{i}\right)$ as:

$$
f_{k}\left(y_{i}, w_{i}\right)=\left|\frac{y_{i}}{\alpha_{k}}\right|^{\beta_{k}}-\left|\frac{y_{i}-w_{i}}{\alpha_{k}}\right|^{\beta_{k}} .
$$

These statistics are used in (2) to generate the randomized test statistic. For a randomly selected collection of subsets $\left\{\Omega_{1}, \ldots, \Omega_{K}\right\}$, the detector computes the detection statistic

$$
T(\mathbf{Y}, \mathbf{w} \mid \Omega)=\sum_{k=1}^{K} \sum_{i \in \Omega_{k}}\left|\frac{Y_{i}}{\alpha_{k}}\right|^{\beta_{k}}-\left|\frac{Y_{i}-w_{i}}{\alpha_{k}}\right|^{\beta_{k}}
$$

and compares it with the threshold $\tau$ in order to make a decision.

Substituting (31) into (23), we obtain the tightest upper bound on $P_{e}$ as

$$
P_{e} \leq \exp \left\{n \sum_{k=1}^{K} \frac{\lambda_{k}}{2}\left(\ln \mathbb{E}\left[\exp s^{*}\left(\left|\frac{X_{1}}{\alpha_{k}}\right|^{\beta_{k}}-\left|\frac{X_{1}-1}{\alpha_{k}}\right|^{\beta_{k}}\right)\right]+\ln \mathbb{E}\left[\exp s^{*}\left(\left|\frac{X_{1}}{\alpha_{k}}\right|^{\beta_{k}}-\left|\frac{X_{1}+1}{\alpha_{k}}\right|^{\beta_{k}}\right)\right]\right)\right\} .
$$

For illustration, let us consider an example (Please refer to Figure 2). Let $p(x)=\frac{1}{4} e^{-\frac{|x|}{2}}$, i.e. the image samples are assumed to be iid Laplacian with variance 8 and mean 0 . The variance of each distribution $q_{k}(\cdot)$ is also 8 if we set

$$
\alpha_{k}=\sqrt{8 \frac{\Gamma\left(\frac{1}{\beta_{k}}\right)}{\Gamma\left(\frac{3}{\beta_{k}}\right)}}
$$




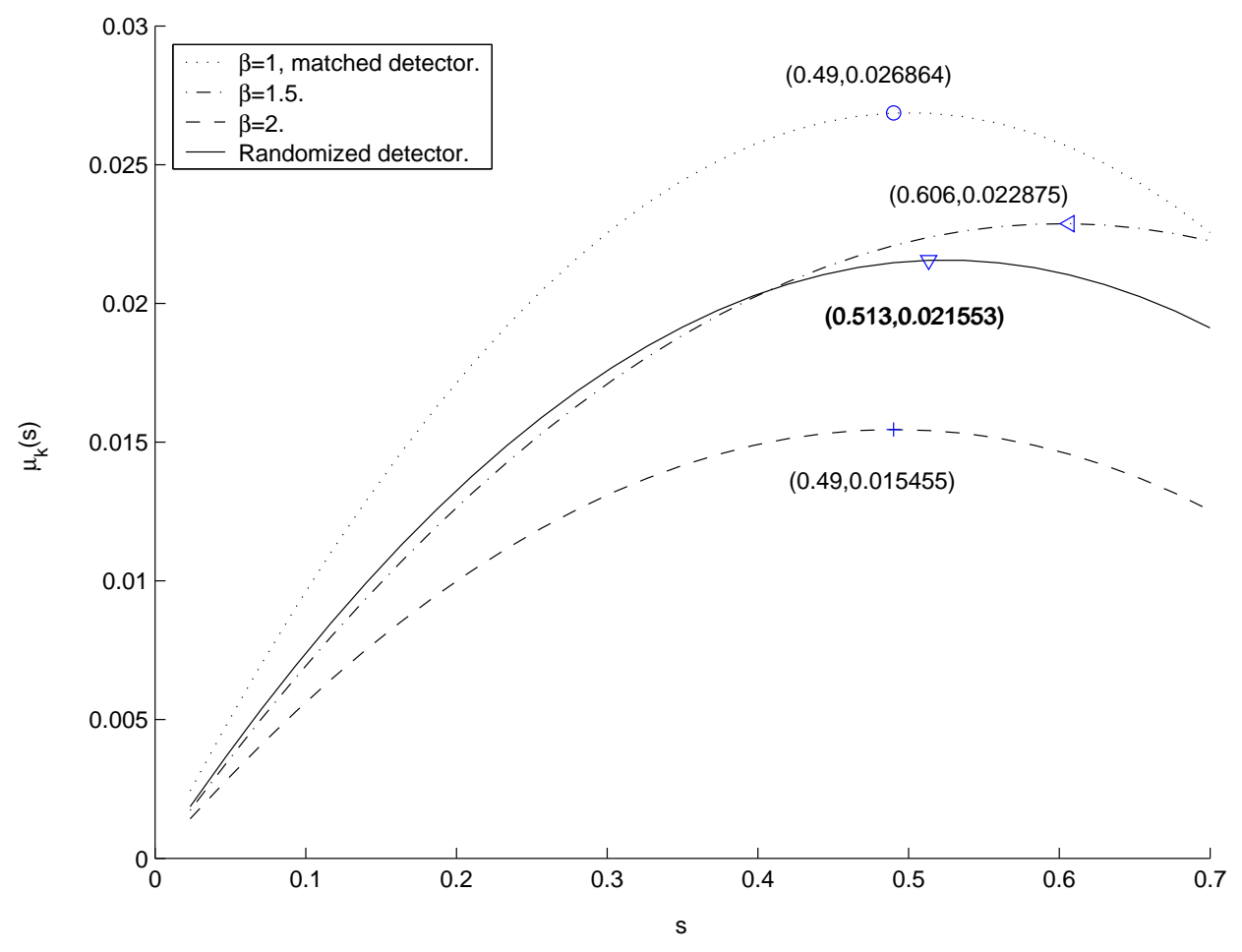

Figure 2. An illustration of our randomized detector, a mixture of three test statistics.

We generate three functions $\left\{f_{k}\right\}$ corresponding to the following three values of $\beta_{k}: 1,1.5$, and 2 . The matched $\log$ likelihood ratio test corresponding to $\beta_{1}=1$ results in the largest exponent $\mu_{1}^{*}=0.026864$, i.e. the minimum probability of error. This is expected since this is the matched detector, i.e. the optimal one. Conversely, the detection test corresponding to $\beta_{3}=2$ results with the smallest, i.e. the "worst", exponent $\mu_{3}^{*}=0.015455$. For $\beta_{2}=1.5$, the error exponent is $\mu_{2}^{*}=0.022875$. For our randomized test, we chose a uniform distribution $\lambda=\left\{\lambda_{k}=\frac{1}{3}, 1 \leq k \leq 3\right\}$. The error exponent for our randomized test is $\mu^{*}=0.021553$, between the previously stated "best" and "worst" exponents, and not very far from our upper bound $\sum_{k=1}^{n} \frac{1}{3} \mu_{k}^{*}=0.021732$.

\section{CONCLUSION}

In this paper, we considered the problem of vulnerability of SS techniques. By introducing randomization into the detector, we hope to increase security. However, the security enhancement does not come for free, but on the contrary at the expense of detection reliability. We showed that by carefully selecting the randomized test statistic as a mixture of "good" statistics, the loss in detection performance can be controlled. We provided the necessary and sufficient condition for exponential decay of the average probability of error, in addition to upper and lower bounds on the exponent of decay. Finally, we applied our results to the class of image signals.

\section{ACKNOWLEDGMENTS}

This work was supported by NSF under grant CCR 03-25924.

\section{REFERENCES}

1. I. J. Cox, M. L. Miller, and J. A. Bloom, Digital Watermarking. San Fransisco: Morgan Kaufmann, 2001.

2. I. J. Cox and J. P. M. G. Linnartz, "Public watermarks and resistance to tampering," in Proc. International Conference on Image Processing (ICIP), only CD version of proceedings available, Santa Barbara, CA, 1997. 
3. M. El Choubassi and P. Moulin, "A new sensitivity analysis attack," in Proc. SPIE Conf., San Jose, CA, January 2005, pp. 734-745.

4. T. Kalker, J. P. Linnartz, and M. van Dijk, "Watermark estimation through detector analysis," in Proc. International Conference on Image Processing (ICIP), vol. 1, pp. 425-429, Chicago, IL, October 1998.

5. J. P. Linnartz and M. van Dijk, "Analysis of the sensitivity attack against electronic watermarks in images," in Proceedings of the Workshop of Information Hiding, Portland, OR, April 1998, pp. 258-272.

6. A. Tewfik and M. Mansour, "LMS-based attack on watermark public detectors," in Proc. IEEE International Conference on Image Processing (ICIP), Rochester, NY, September 2002, pp. 649-652.

7. P. Comesãna, L. Pérez-Freire, and F. Pérez-Gonzaléz, "The return of the sensitivity attack," in Proc. IWDW Conf., Siena, Italy, September, 2005, pp. 260-274.

8. R. Venkatesan and M.H. Jakubowski, "Randomized Detection For Spread-Spectrum Watermarking: Defending Against Sensitivity and Other Attacks," Proc. ICASSP Conf., Philadelphia PA, March 2005.

9. H.V. Poor, An Introduction to Signal Detection and Estimation, Springer-Verlag, New York, 1988.

10. D. Kazakos, "Signal Detection Under Mismatch", IEEE Transactions On Information Theory, Vol. IT-28, July 1982, pp. 681-684.

11. M. Barni, F. Bartolini, A. Piva, and F. Rigacci, "Statistical modeling of full frame DCT coefficients", in Proceedings of the 9th European Signal Processing Conference (EUSIPCO), Rhodes, Greece, 1998, pp. 1513-1516. 